\title{
Estimation of the Operating Parameters of Miniature Radioisotope Thermoelectric Power Unit Based on the Th-228 Isotope
}

\section{V. Fetisov'1, O. S. Vasilyev¹, P. V. Borisyuk ${ }^{1}$, and Yu. Yu. Lebedinskiii,1,2}

${ }^{1}$ National Research Nuclear University MEPhl (Moscow Engineering Physics Institute), Kashirskoe sh. 31, 115409, Moscow, Russia

${ }^{2}$ Moscow Institute of Physics and Technology (State University), Institutskiy per. 9, 141701, Dolgoprudny, Moscow Region, Russia

\section{Abstract}

The article considers the construction of a miniature radioisotope power unit based on thermoelectric conversion of thermal energy released during nuclear decay. It is proposed to use thin fluoropolymer films (membranes) as a dielectric heat-insulating material. The results of numerical simulation of a prototype of a miniature radioisotope

Corresponding Author: V. V. Fetisov vvfetisov@mephi.ru

Received: 22 July 2018 Accepted: 9 September 2018 Published: 8 October 2018

Publishing services provided by Knowledge E

(c) V. V. Fetisov et al. This article is distributed under the terms of the Creative Commons

Attribution License, which permits unrestricted use and redistribution provided that the original author and source are credited.

Selection and Peer-review under the responsibility of the Breakthrough Directions of Scientific Research at MEPhI Conference Committee. thermoelectric battery unit based on the thorium-228 isotope in the ANSYS program are presented. The geometry of the system has been optimized. It was established that the temperature of the source can reach about $1033 \mathrm{~K}$, and the efficiency of the considered battery unit can reach $16.8 \%$, which corresponds to modern power supplies of this type.

\section{Introduction}

The development of nanoelectronics, in particular, micro- and nanoelectromechanical systems (MEMS and NEMS) [1] is currently limited by the lack of effective commensurate power supplies [2]. Power sources based on radioisotope source are one of the perspective miniature power supply units for MEMS and NEMS [3]. Their advantages include long, stable work, lack of maintenance, noiselessness. One of the shortest ways to convert the energy of nuclear decay into electrical one is the thermoelectric conversion [4]. So, it is necessary to consider the process of conversion of the thermal energy of a radioisotope into electrical one at the characteristic scales of the system in order to prevent energy losses.

In this article, the possibility of creating miniature thermoelectric radioisotope battery is considered. The design of the power source is shown in Figure $1(\mathrm{a}, \mathrm{b})$.

In the figure, the element of a disk form (1) located in the center of the upper surface S OPEN ACCESS of the unit represents the radioisotope source of thermal energy, that initiates local 
heating of the junction point of a pair of thermoelectric material tracks (2), (3). The thermal insulation layer (4) reduces the energy loss on the substrate (5) that operates as the thermostat. The substrate has a through hole, which is necessary for a high thermal insulation of the system. When using this type of power source design, a number of requirements on the geometry and materials arise. All of them are described in this article.

(a)

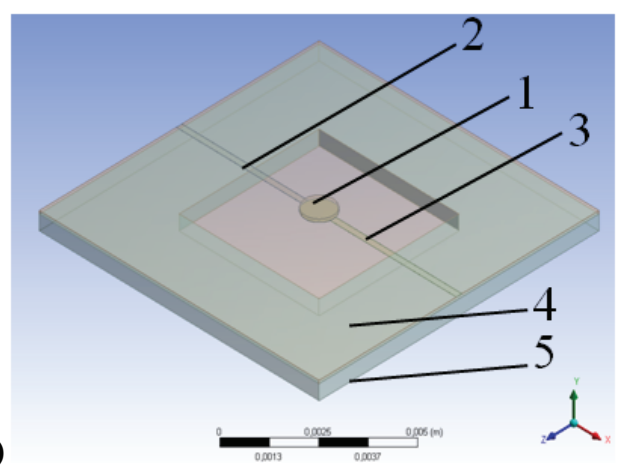

(b)

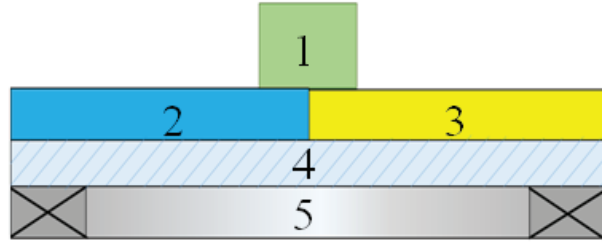

Figure 1: (a) Isometric view of the construction of a radioisotope thermoelectric power source; (b) Schematic representation of the design of a radioisotope thermoelectric power source (side view of a section) (not in scale).

\section{Materials and Experimental Procedure}

Let us consider in detail all the elements of the nuclear power source design shown in Figure 1. A radioisotope that produces $\alpha$ - or $\beta$-particles with a high specific power is needed for use as a fuel element in a miniaturized power source. The lifetime of the radioisotope is an important criterion here. Typically, the specific power is inversely proportional to the lifetime, that is, the higher the value of the half-life is, the lower is the specific power of the material. A pure $\alpha$-source of polonium-210 has high specific power $(N=210 \mathrm{~W} / \mathrm{g})$ and short half-life $\left(T_{1 / 2}=134\right.$ days), so it is convenient to use for energy-intensive but not time-consuming tasks. In turn, such element as nickel63 ( $\beta$-decay, $N=0.5 \mathrm{~W} / \mathrm{g}, T_{1 / 2}=100.1$ years) is suitable for long life system supplying, that doesn't require high electrical power. In laboratory conditions, it is convenient to use a radioisotope that has parameters of specific power and lifetime, such as thorium-228 has ( $\alpha$-decay, $N=26 \mathrm{~W} / \mathrm{g}, T_{1 / 2}=1.9$ years). It should be noted that metallic thorium is hardly accessible, therefore, it is advisable to use thorium dioxide, which is easy to synthesize and, in addition, is much less susceptible to self-destruction and self-dispersion. To ensure maximum heating of nuclear material and minimize heat losses, the radioisotope source and hot ends of thermoelectric materials are 
located on a thin membrane of the heat insulator. Calculations show that silicon dioxide $\mathrm{SiO}_{2}$ thermal insulation membrane with a thermal conductivity of $k=1.5 \mathrm{~W} /(\mathrm{m} \cdot \mathrm{K})$ the effective thickness is about 1-5 $\mathrm{mm}$. At the same time, with the use of PVdF (thermal conductivity $k=0.2 \mathrm{~W} /(\mathrm{m} \cdot \mathrm{K}))$ as thermal insulation material, the efficiency of the radioisotope/membrane system is significantly increased. We have developed a technique for depositing thin PVdF films and membranes on $\mathrm{SiO}_{2} / \mathrm{Si}(001)$ substrates of $1 \mathrm{X} 1 \mathrm{~cm}$ in size with a central through hole of $6 \mathrm{~mm}$ in diameter. Deposition process was the following: the pre-cleaned substrates were placed into a Petri dish and poured out of $5 \%$ acetone PVdF solution and then the Petri dish was closed with a lid and held for 20 hours until completely dried. This method provides formation of plain and smooth heat-insulating membranes with an average thickness of $\sim 20 \mu \mathrm{m}$. The SEM image of such film is shown in Figure 2(a).

Figure 2(b) shows a SEM image of a similar PVdF membrane with a thermoelectric material deposited onto its surface in the form of a track with dimensions of $8 \times 0.5 \times$ 0.001 (length $\times$ width $\times$ height, $\mathrm{mm}$ ).

The geometry of thermoelectric tracks was selected from the following considerations. The peak electrical power of the power unit is determined by the short-circuit current $I_{s c}$ multiplied by the open-circuit power supply voltage $U$. The value of $U$ in this case is determined by the contact potential difference (Seebeck effect) and, consequently, depends on the temperature gradient along the thermoelectric tracks. It is important to note that the temperature gradient, in turn, depends on the cross-section of the thermoelectric track s. So the smaller the cross-section of the thermoelectric track is, the higher the temperature can be obtained. Heat transfer in metals is carried to the greatest extent by the flux of free electrons, therefore, the maximum current of the power source will be proportional to the heat fluxes $\left(\mathrm{W} / \mathrm{cm}^{2}\right)$ passing along the thermoelectric tracks multiplied by the cross-section. Thus, we can introduce the parameter $\alpha$, which is obtained by multiplying the heat fluxes by the temperature difference between the hot and cold ends of thermoelectric tracks. In this case, the parameter $\alpha$ is a function that depends on the cross-section of thermoelectric tracks, and determines the efficiency of the power source. As the cross-section decreases, the temperature of the hot end will increase, but the heat flux will decrease, so if we find the maximum of the function of parameter $\alpha$, we will be able to determine uniquely the optimal geometry of the system. 

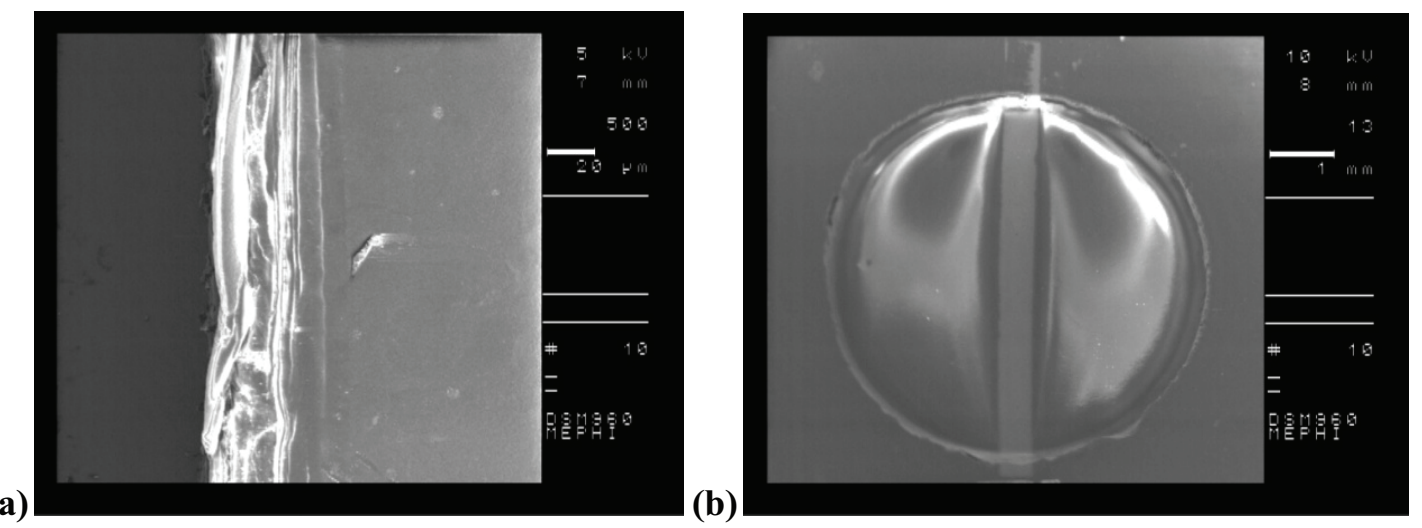

Figure 2: (a) SEM image of a PVdF film on silicon substrate (side view); (b) SEM image of gold thermoelectric track on the PVdF membrane.

\section{Results}

A number of model experiments were carried out in the software package ANSYS, in which the temperature and heat flux distributions along thermoelectric tracks were found for different cross-sections. For each calculation, the geometric parameters and materials of the radioisotope element and heat insulator remained unchanged, but the width and height of thermoelectric tracks varied (Table 1).

TABLE 1: Geometric and thermophysical parameters of the elements of the construction of a radioisotope thermoelectric power source.

\begin{tabular}{|c|c|}
\hline \multicolumn{2}{|l|}{ Substrate characteristics } \\
\hline Length $\times$ width $\times$ height, $\mathrm{cm} \times \mathrm{cm} \times \mathrm{cm}$ & $1 \times 1 \times 0.05$ \\
\hline Hole diameter, cm & 0.6 \\
\hline Thermal conductivity (Si), W/(mK) & 148 \\
\hline \multicolumn{2}{|l|}{ Characteristics of the thermal insulation layer } \\
\hline Length $\times$ width $\times$ height, $\mathrm{cm} \times \mathrm{cm} \times \mathrm{cm}$ & $1 \times 1 \times 0.002$ \\
\hline Thermal conductivity $(\mathrm{PVdF}), \mathrm{W} /(\mathrm{m} \cdot \mathrm{K})$ & 0.2 \\
\hline \multicolumn{2}{|l|}{ Characteristics of thermoelectric tracks } \\
\hline Width, $\mu \mathrm{m}$ & $100 \div 500$ \\
\hline Height, $\mu \mathrm{m}$ & $10 \div 100(10)$ \\
\hline Thermal conductivity (Au), w/(mK) & 204 \\
\hline Thermal conductivity (Pt), W/(mK) & 72 \\
\hline \multicolumn{2}{|l|}{ Characteristics of the radioisotope source } \\
\hline Radius, $\mu \mathrm{m}$ & 500 \\
\hline Height, $\mu \mathrm{m}$ & 10 \\
\hline Материал & $\mathrm{ThO}_{2}$ (Thoriumdioxide-228) \\
\hline Specific power, W/cm³ & 221.7 \\
\hline Power, mV & 17.8 \\
\hline Thermal conductivity, W/(mK) & 9.7 \\
\hline
\end{tabular}


The analysis of the obtained data resulted in the dependence of the parameter $\alpha$ on the cross-section of thermoelectric tracks. For convenience, the heat fluxes were normalized to the total power released from the nuclear element. The plot of the parameter $\alpha$ is shown in Figure 3.

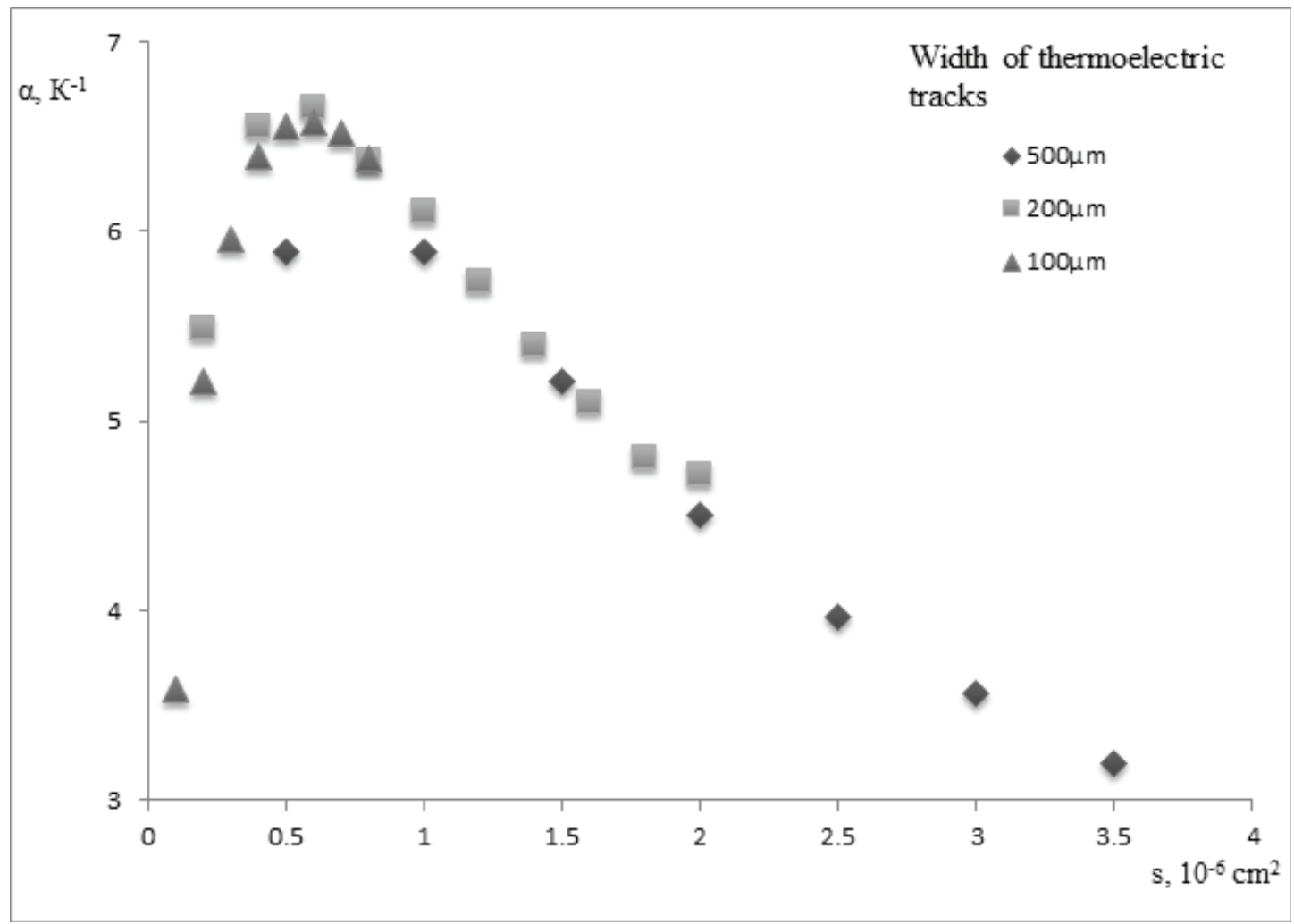

Figure 3: Dependence of the parameter $\alpha\left(\mathrm{K}^{-1}\right)$ on the cross-section of thermoelectric tracks $\left(10^{-6} \mathrm{~cm}^{2}\right)$.

The graph has a bell shape. One can clearly see the maximum of the function $\alpha$, which is achieved at a cross-section of $0.6 \times 10^{-6} \mathrm{~cm}^{2}$. At this point, the temperature of the hot end is approximately $1033 \mathrm{~K}$. That cross-section of thermoelectric track is the optimal one. These calculations were carried out for metallic gold and platinum materials of the thermoelectric tracks. Such materials were chosen because gold and platinum are two noble metals with thermoelectric power value of a different sign. However, it must be said that these materials are suitable only for testing the technique. Nevertheless, even for a such test materials the efficiency of such radioisotope thermoelectric power unit can be compared with modern power units of this type. Indeed, the efficiency of thermoelectric materials is usually described by the $Z T$ factor, which is directly proportional to the square of the Seebeck coefficient $S^{2}$, the electrical conductivity of the material $\sigma$, and inversely proportional to the thermal conductivity $\mathrm{K}$

$$
Z T=T \frac{S^{2} \sigma}{\kappa} .
$$


In order to make this parameter higher, our scientific group suggests using porous films made of metal nanoclusters as thermoelectric materials [5]. It is expected that the porous structure of the material will lead to a decrease of the phonon thermal conductivity due to the scattering of phonons at the interfaces of the nanoclusters. In case of metal porous film, the electrical conductivity will be comparable with the metallic one due to electron tunneling between nanoclusters and percolation effects. A significant increase of the Seebeck coefficient is also expected [6].

The principle of operation of thermoelectric materials is similar to the principle of operation of a thermal engine that uses electrons and positively charged holes as energy carriers. The efficiency of the thermoelectric conversion is determined by the Carnot cycle and the material factor $Z T$ [7]. The value of the efficiency of thermoelectric converters in can be found by the formula:

$$
=\frac{\Delta T}{T_{h}}\left(\frac{\sqrt{1+Z \bar{T}}-1}{\sqrt{1+Z \bar{T}}+\frac{T_{c}}{T_{h}}}\right),
$$

where $\bar{T}=\frac{T_{h}-T_{c}}{2}$ and $\Delta T=T_{h}-T_{c}, T_{h} \mathrm{n} T_{c}$ - temperature of hot and cold ends of thermoelectric tracks, respectively.

The figure of merit of modern thermoelectric materials at the operating temperature of about $1064 \mathrm{~K}$ is equal to unity $Z T=1$ [8]. Using this value for calculation of the efficiency of a miniature radioisotope thermoelectric battery, one can get the estimation of the highest possible efficiency. In the aforementioned model, the temperature of the cold and hot ends of thermoelectric tracks is $22^{\circ} \mathrm{C}(295 \mathrm{~K})$ and $760^{\circ} \mathrm{C}(1033 \mathrm{~K})$, respectively. Then, the obtained value of the efficiency of a radioisotope thermoelectric power source is $n=16.8 \%$. Considering that the geometry of the structure was chosen optimally, one can say that almost all the energy of the fuel element is transferred by the thermoelectric materials. Therefore, it is possible to multiply the total thermal power of a radioisotope source $(N=17.8 \mathrm{~mW})$ by the obtained value of the efficiency and estimate the electric power of the battery, which in this case will be $2.9 \mathrm{~mW}$.

\section{Conclusion}

In this article, one of the design solutions for creating a miniature radioisotope thermoelectric power source is considered. Materials of structural elements (thorium-228, PVdF, nanocluster porous films) were selected. A technique for the thermoelectric tracks geometry optimization using the parameter $\alpha$ was developed. The estimation 
(the highest possible value for the system considered) of the efficiency of the source ( $\eta=16.8 \%$ ) was used to find the electric power that occurred to be $N_{e}=2.9 \mathrm{~mW}$.

\section{Funding}

This work was financially supported by the Grant of the Russian Science Foundation (Project No. 16-19-00168).

\section{References}

[1] Kim, K., Guo, J., Xu, X., et al. (2015). Micromotors with step-motor characteristics by controlled magnetic interactions among assembled components. ACS Nano, vol. 9, pp. 548-554.

[2] Holmberg, S., Perebikovsky, A., Kulinsky, L., et al. (2014). 3-D micro and nano technologies for improvements in electrochemical power devices. Micromachines, vol. 5, pp. 171-203.

[3] Lal, A. and Blanchard, J. (2004). Daintiest dynamos [nuclear microbatteries]. IEEE Spectrum, vol. 41, pp. 36-41.

[4] Prelas, M. A., Weaver, C. L., Watermann, M. L., et al. (2014). A review of nuclear batteries. Progress in Nuclear Energy, vol. 75, pp. 117-148.

[5] Borisyuk, P. V., Vasilyev, O. S., Krasavin, A. V., et al. (2016). Nanocluster metal films as thermoelectric material for radioisotope mini battery unit. Chemical Physics, vol. 478, pp. 2-7.

[6] Ziman, J. M. (1961). The ordinary transport properties of the noble metals. Advances in Physics, vol. 10, pp. 1-56.

[7] Macia-Barber, E. (2015). Thermoelectric Materials Advances and Applications. Boca Raton: Taylor \& Francis Group.

[8] Gayner, C. and Kar, K. (2016). Recent advances in thermoelectric materials. Progress in Materials Science, vol. 83, pp. 330-82. 